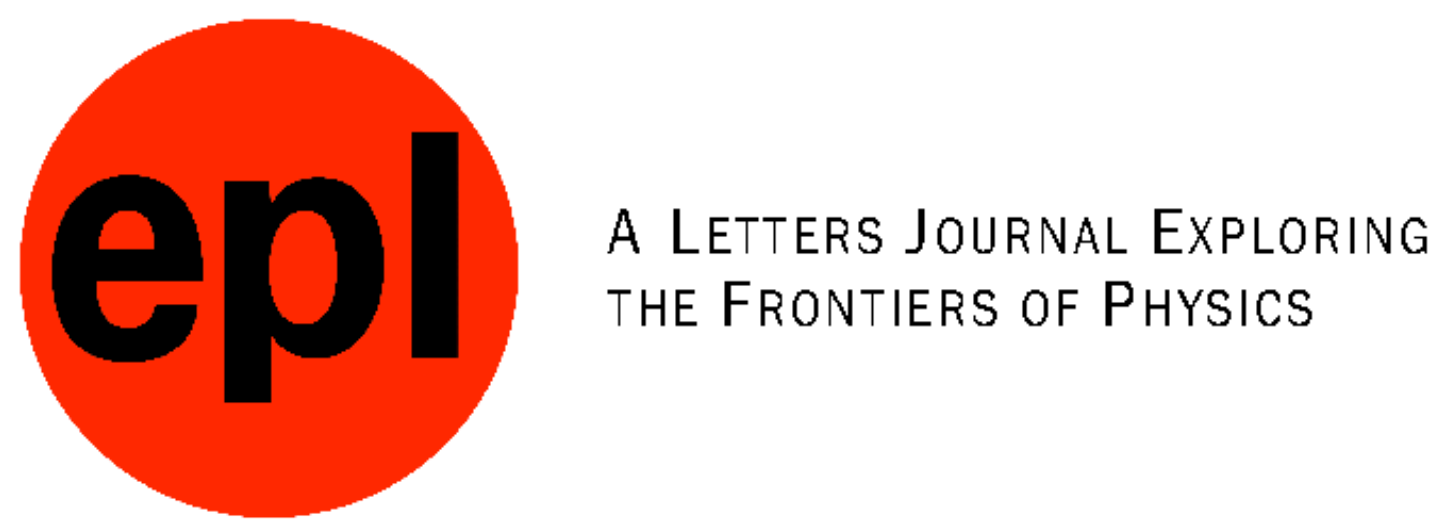

OFFPRINT

\title{
Microstructure-induced giant elastic nonlinearity of threshold origin: Mechanism and experimental demonstration
}

V. Yu. Zaitsev, A. Dyskin, E. Pasternak and L. Matveev EPL, 86 (2009) 44005

Please visit the new website www.epljournal.org 


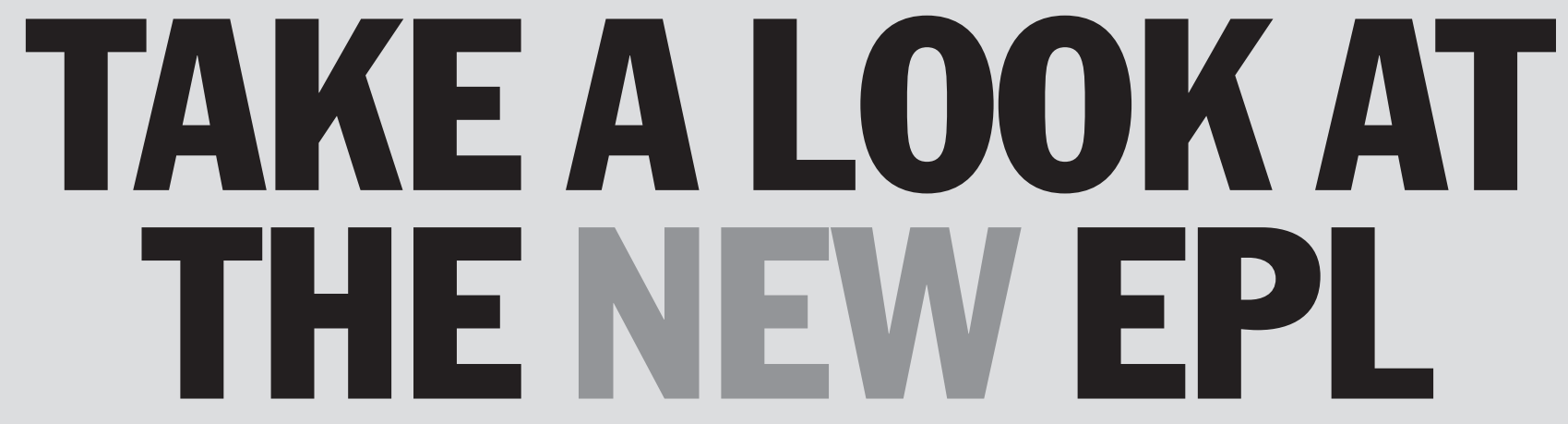

\section{Europhysics Letters (EPL) has a new online home at www.epljournal.org}

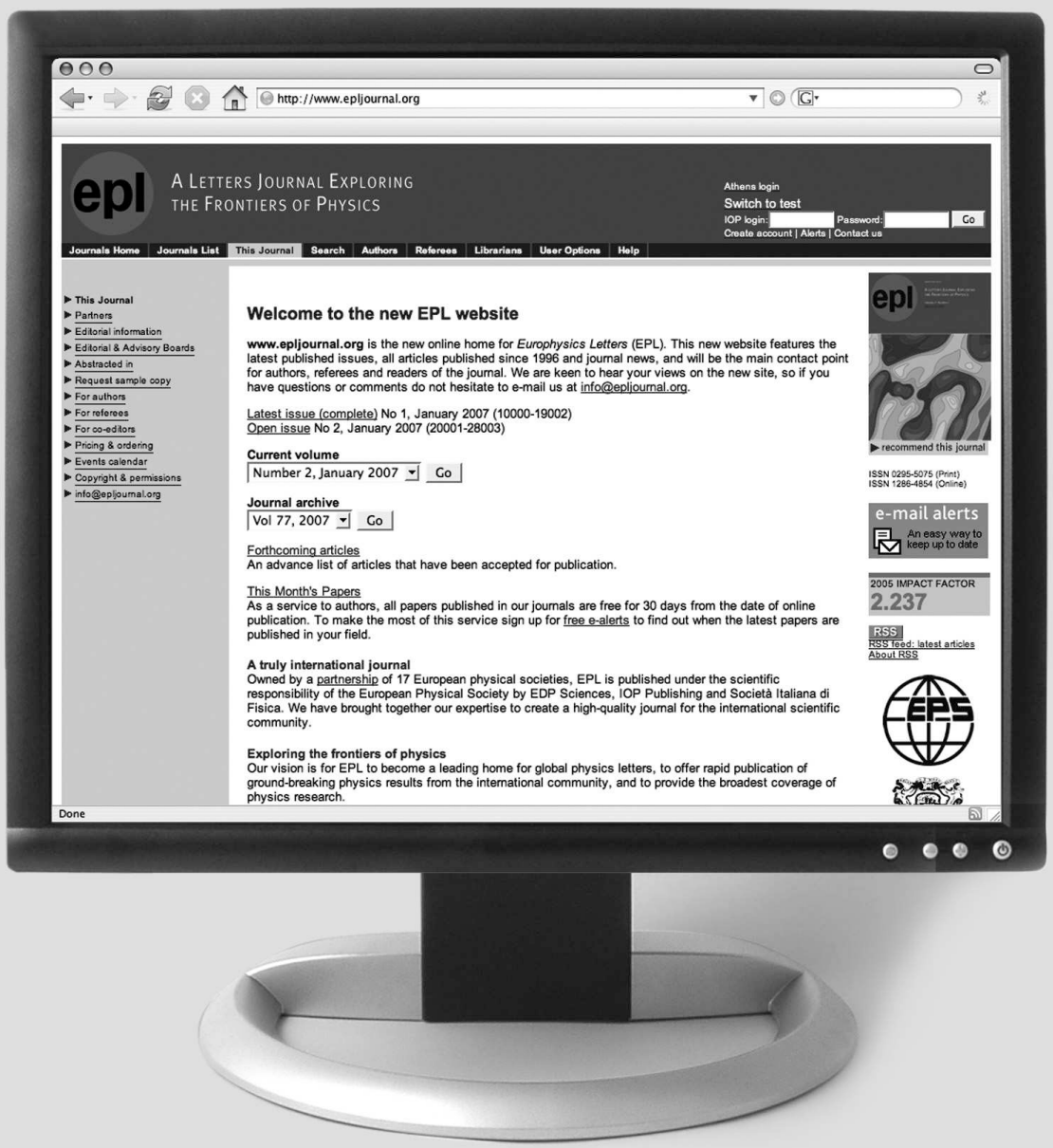

Take a look for the latest journal news and information on:

- reading the latest articles, free!

- receiving free e-mail alerts

- submitting your work to EPL 


\title{
Microstructure-induced giant elastic nonlinearity of threshold origin: Mechanism and experimental demonstration
}

\author{
V. Yu. Zaitsev ${ }^{1(a)}$, A. Dyskin ${ }^{2}$, E. Pasternak ${ }^{2}$ and L. Matveev ${ }^{1}$ \\ ${ }^{1}$ Institute Applied Physics, Russian Academy of Sciences - Uljanova St. 46, 603950, Nizhny Novgorod, Russia \\ ${ }^{2}$ University of Western Australia - 35 Stirling Hwy, Crawley, WA, 6009, Australia
}

received 8 March 2009; accepted in final form 29 April 2009

published online 29 May 2009

PACS 43.25.+y - Nonlinear acoustics

PACS $43.25 . \mathrm{Ba}$ - Parameters of nonlinearity of the medium

PACS 83.60.Df - Nonlinear viscoelasticity

\begin{abstract}
There are known numerous examples of strong physical nonlinearity of elastic microinhomogeneous materials caused by the presence of a small amount of a "soft" component (cracks, intergrain contacts, bubbles, etc.). The dimensionless parameter $B / A$ of quadratic nonlinearity for such materials can be on the order of $10^{2}-10^{3}$ in contrast to values on the order of unity typical of ideal crystals, homogeneous amorphous solids or liquids. The sign of this nonlinearity is such that all those materials become stiffer as pressure increases. We consider an extension of this "soft-rigid paradigm" of the nonlinearity increase by accounting for threshold-type properties intrinsic to some inclusions. We show that the nonlinearity in this case can additionally be orders of magnitude higher. We present an example of such a material which unlike "normal" media becomes softer with increasing pressure. Its quasistatic negative parameter $B / A$ reaches $\sim(0.5-1) \cdot 10^{5}$, which to our knowledge is a record value.
\end{abstract}

Copyright (C) EPLA, 2009

Introduction. - It is well known that many microstructure inhomogeneous elastic materials can exhibit pronounced nonlinearity even at fairly small acoustic strains $\left(\varepsilon \lesssim 10^{-6}-10^{-5}\right)$ for which homogeneous materials obey the linear Hooke law with a high accuracy [1]. This difference is due to the presence of certain structural features with high contrasting "softness" as compared to the matrix material. Local strains at these structural features can be orders of magnitude greater than the average strain. Thus, the material behaviour may become strongly nonlinear even at small concentrations of the soft inclusions $[2,3]$. This mechanism of nonlinearity is sometimes called the "soft-rigid paradigm" [4]. In solids, the role of such soft inclusions is played, first of all, by crack-like defects and intergrain contacts. Additional factors, e.g., microfriction and/or adhesion phenomena [1] at the contacts or their clapping can make this nonlinearity nonanalytic. For example, "clapping" nonlinearity of Hertzian contacts is diod like with local stress proportional to the power $3 / 2$ of strain $[5,6]$. This makes it impossible to approximate the stress-strain relationship $\sigma(\varepsilon)$ by a power series expansion

\footnotetext{
(a) E-mail: vyuzai@hydro.appl.sci-nnov.ru
}

$\sigma(\varepsilon)=M\left(\varepsilon+\beta \varepsilon^{2} / 2+\ldots\right)$, where $\beta$ is the dimensionless quadratic nonlinearity parameter (often denoted as $B / A$ in acoustics). Nevertheless, even for nonanalytic nonlinearities, quite often "equivalent" quadratic or cubic nonlinearity parameters are used in order to compare the levels of the microstructural and conventional atomic nonlinearities. However, such equivalent representations do not ensure the correct functional description of nonlinear effects and their extrapolation to other strain levels. For instance, if the actual dependence is different from quadratic, the equivalent representation can result in strong overestimation of the actual nonlinear parameter (e.g. for strains $\varepsilon \sim 10^{-6}$, the $\varepsilon^{3 / 2}$-case mentioned above differs $\varepsilon^{-1 / 2}=10^{3}$ times from $\varepsilon^{2}$ ).

In this paper, we draw the attention to an important (but usually disregarded) feature intrinsic to some "soft" microstructural features - the threshold-type response to applied pressure (stress). We show that this feature can lead to additional (orders of magnitude) increase in the microstructure-induced nonlinearity compared to the conventional $[2,4]$ soft-rigid mechanism. Finally, we present an example of a material in which this thresholdtype mechanism is implemented and which demonstrates a genuine (i.e., not equivalent) quadratic-nonlinearity 
parameter of the order of fifty thousands, which to the best of our knowledge is a record value.

Hollow shells as an example of thresholdbehaving objects. - An important and historically first well-understood example of the manifestation of the soft-rigid mechanism is the case of liquids with gas bubbles. Quite small amounts of bubbles strongly reduce the sound velocity and increase nonlinearity of the liquid due to high contrast between the compressibility of the gaseous and liquid phases $[7,8]$. In solids the situation is normally different. Since the shear modulus is no longer negligible, pores can stand on their own without internal gas pressure. Typically, the effective compressibility of the pores is not much higher than that of the matrix, hence small concentrations of the pores do not much increase the deformability of the material. An exception is the class of so-called nearly incompressible materials, for which the ratio of the shear Lamé parameter to the compressional one is low, $\mu / \lambda \ll 1$ (i.e., the Poisson's ratio is close to 0.5 like in liquids). In such materials, the hoop stiffness of the pores is strongly reduced. As a result, even at low densities the pores can markedly reduce the macroscopic bulk modulus and increase the elastic nonlinearity of the material [9]. In addition, highly compressible pores produce strong scattering of acoustic waves. The latter suggested the idea to use bubble-like inclusions as a contrast agent in medical ultrasound diagnostics (e.g., for better visualization of blood vessels in tissues). To avoid undesirable biological effects and to ensure stable and controllable "bubbles", special contrast agents representing thin plastic gas-filled shells of a micrometer-scale size were developed [10]. Similar microspheres are produced for engineering purposes (e.g., as filling substances in paints, etc.) [11].

Despite the obvious similarity, these specially encapsulated bubbles mechanically strongly differ from the conventional ones due to the presence of an elastic shell. The effect of their presence in the material in a certain sense can be even opposite to the effect of bubbles or pores. Because of the hoop stiffness of the shell, the compressibility of such encapsulated pores or bubbles can be much smaller than the compressibility of a genuine bubble or shell-less pore of the same size. This situation, however, only lasts until the differential pressure reaches a threshold (critical) value corresponding to the shell buckling [12],

$$
p_{c r}=\frac{2 E_{s} t^{2}}{R^{2} \sqrt{3\left(1-\nu^{2}\right)}},
$$

where $E_{s}$ is the Young modulus of the shell material, $\nu$ is its Poisson's ratio, and $t$ and $R$ are its thickness and radius, respectively. For pressures $p>p_{c r}$, the shell loses its stability and buckles, so that its compressibility drastically increases and becomes close to that of a shellless gas bubble or pore (i.e. $10^{4}-10^{5}$ times higher than for the matrix material), whereas before buckling the value of the effective compression modulus $K_{s}=2 E_{s} t /[3(1-\nu) R]$ of the shell [13] remains many times higher.
This threshold-type behaviour of encapsulated bubbles can drastically alter the pressure dependence of the compressibility. For liquids and incompressible materials with conventional bubbles and pores, their compressibility decreases with increasing pressure (since the total volume of the bubbles decreases and the adiabatic compressibility of bubbles inversely proportional to the gas pressure simultaneously decreases). In contrast, for materials containing microspheres with different buckling thresholds, the compressibility can gradually increase with increasing pressure. Indeed, as the pressure increases, the sequential buckling of the microspheres results in the increase in the content of highly compressible fraction in the material, which causes decrease in the sound velocity $c(p)$. Therefore, the signs of the derivatives $\mathrm{d} c / \mathrm{d} p$ and $\mathrm{d} K / \mathrm{d} p$ (or, what is called the nonlinearity sign) can be opposite for the encapsulated and shell-less bubbles. Similar qualitative arguments were put forward in [14], where the negative $\mathrm{d} c / \mathrm{d} p$ was experimentally demonstrated for a mixture of hollow microspheres with castor oil.

It should be noted, however, that after buckling, the shells behave like conventional pores or bubbles and give a strong positive contribution to $\mathrm{d} c / \mathrm{d} p$. The negative $\mathrm{d} c / \mathrm{d} p$ can only occur if the effect of the gradual threshold buckling dominates. In what follows, in the development of the analysis $[9,15,16]$ of conventional bubble-like voids, we analyse the effect of the threshold behaviour of encapsulated bubbles, derive expressions for the material nonlinearity parameter and formulate the criteria of either positive or negative sign of the nonlinearity for liquids and incompressible elastic materials containing encapsulated bubbles and pore. Experimental evidence of strong dominance of the threshold-type structural mechanism over the soft-rigid one is also presented.

Model of elastic nonlinearity of incompressible material with hollow microspheres. - For materials with $\mu \ll \lambda$ (for which Poisson's ratio $\nu=\lambda /[2(\lambda+\mu)] \rightarrow$ $0.5)$, the modulus $M=\lambda+2 \mu$ which controls the pressurewave velocity and the bulk modulus $K=\lambda+2 \mu / 3$ are practically equal $(M \simeq K \simeq \lambda)$. Following [1,9] we also use the fact that in materials with $\mu \ll \lambda$, the stress state created by a compressional wave with a high accuracy can be considered as almost hydrostatic pressure $p$.

We assume that the unit volume of the material contains encapsulated spherical pores with the distribution function $n(r)$ over their radii. The interaction between the inclusions can be neglected due to their low concentration. A part of them is buckled at the current equilibrium pressure $p$ and their relative volume content is

$$
v(p)=\int_{r\left(p=p_{c r}\right)}^{\infty}\left(4 \pi R^{3} / 3\right) n(R) \mathrm{d} R
$$

where $4 \pi R^{3} / 3$ is the initial volume of a single microsphere. We recall that the contribution of unbuckled microspheres 
(for which $p<p_{c r}$ ) to the material compressibility is negligible compared to that of buckled microspheres (for which $p>p_{c r}$ ). We also make the assumption that since the microspheres are usually gas filled (e.g., at an atmospheric pressure), their volume just after buckling remains approximately the same as it was at the buckling threshold. Indeed, for typical properties of real gas-filled microspheres (e.g. [11]) with radius $r=20-40 \mu \mathrm{m}$ and the characteristic wall thickness $t=0.1 \mu \mathrm{m}$, the critical pressure $p_{c r}$ (see eq. (1)) is $\sim 10^{4} \mathrm{~Pa}$, which is only $10 \%$ of the atmospheric pressure of the gas in the shell. Therefore, immediately after the bucking threshold, the main effect of buckling is the drastic jump in the microsphere compressibility rather than the change in its volume.

To determine the effective bulk modulus, following $[9,16]$ consider a thin layer with thickness $\Delta x$ of such an incompressible material containing a large number of buckled microsperes with volume content $v$, subjected to incremental pressure $\mathrm{d} p$ in the field of a compressional wave propagated along the $x$-axis. The corresponding thickness variation $\Delta u$ of the layer can be written as

$$
\Delta u=[\mathrm{d} p / K+\mathrm{d} v] \Delta x
$$

where $\mathrm{d} v$ is the pressure-dependent increment of the volume content of the buckled microspheres. The first term reflects the compressibility of the matrix. Taking into account that for the considered nearly incompressible material with $\mu \ll \lambda$, its relative volume variation almost coincides with strain $(\varepsilon=\Delta u / \Delta x=\mathrm{d} V / V)$, we obtain

$$
\mathrm{d} V / V=\mathrm{d} p / K+\mathrm{d} v \text {. }
$$

Comparing this equation with the definition of the effective compressibility $1 / K_{\text {eff }}=(1 / \mathrm{d} p)(\mathrm{d} V / V)$, one obtains

$$
1 / K_{\text {eff }}=1 / K+\mathrm{d} v / \mathrm{d} p .
$$

To determine the second term in eq. (5), we first note that after buckling (when the microsphere behaves like a "normal" bubble) the volume change $\mathrm{d} V_{R}$ of such a single bubble of radius $R$ caused by the pressure increment $\mathrm{d} p$ can be approximated by analogy with $[1,9,16]$ as

$$
\mathrm{d} V_{R}(p)=g_{1}(R, p) \mathrm{d} p,
$$

where we take into account that the compressibility $g_{1}$ of the void encapsulated in a buckled shell depends on pressure $p$. Generally speaking, this dependence can be related to either adiabatically varying pressure of sufficiently strong waves (as discussed in $[9,16]$ for shell-less pores) or the isothermally varying quasistatic pressure (as in the experiments discussed below). In order to facilitate further comparison with previous results $[1,2,9,16]$ and our own experiments on the pressure dependence of the velocity of a probe wave we concentrate on the case of sufficiently weak sound waves that are only capable of compressing and expanding the already buckled and thus soft microspheres. Such waves do not produce appreciable buckling themselves ( since $\mathrm{d} p \ll p$ ).
Furthermore, they are not able to restore the shape of buckled shells of the microspheres during the rarefaction phase since a shell decreases its volume upon buckling by a small, but finite value, so that the shape restoration requires non-infinitesomal rarefaction amplitudes.

Assuming that the shells have identical wall thickness (so that $p_{c r}$ is determined only by radius $R$ ), we thus can rewrite eq. (5) as

$$
1 / K_{e f f}=1 / K+\int_{r\left(p=p_{c r}\right)}^{\infty}\left[g_{1}(R, p)\right] n(R) \mathrm{d} R .
$$

The key difference of eq. (7) from the similar ones for conventional bubbles and pores $[9,16]$ is that the lower limit in the integral over the distribution function is pressure dependent, which indicates that with increasing pressure, the contribution of the shells to the material compressibility gradually increases. Thus, the pressure dependence of the material compressibility is determined by the interplay of two different factors: the gradually increasing portion of the buckled microspheres with increasing pressure and the pressure dependence of each microsphere volume after its buckling.

Equation (7) can be used to estimate the value of the dimensionless nonlinearity parameter $\beta$ (or $B / A$ in the notations used in [14]) which characterises the dependence of the bulk modulus on the current pressure $p$ :

$$
\beta=\mathrm{d} K_{e f f} / \mathrm{d} p \approx 2 \rho c \mathrm{~d} c / \mathrm{d} p,
$$

where $\rho$ is density. Using eqs. (6) and (7) we obtain

$$
\begin{gathered}
\beta=\mathrm{d} K_{e f f} / \mathrm{d} p=\beta_{c}+\beta_{t h}, \\
\beta_{c}=-K_{e f f}^{2} \int_{r\left(p=p_{c r)}\right.}^{\infty}\left[\mathrm{d} g_{1}(R, p) / \mathrm{d} p\right] n(R) \mathrm{d} R \\
\beta_{t h}=K_{e f f}^{2} \frac{\mathrm{d} R_{c r}(p)}{\mathrm{d} p} g_{1}\left(R_{c r}, p\right) n\left(R_{c r}\right) .
\end{gathered}
$$

Here, $R_{c r}(p)$ is the smallest radius of the shells which buckle under pressure $p$. Assuming that the nonlinearity of the material is estimated by measuring the dependence of the probe-wave velocity as a function of the static pressure, the pressure derivatives in eqs. (8)-(11) correspond to isothermal (slow) pressure variation, whereas ratio $\mathrm{d} v / \mathrm{d} p$ in eq. (5) corresponds to adiabatic variations in the wave field. In eq. (9), the term $\beta_{c}$ is associated with the conventionally discussed soft-rigid mechanism and describes the contribution of soft buckled microspheres to the overall nonlinearity. The term $\beta_{t h}$ appears due to the threshold buckling of the microspheres and describes the corresponding contribution of the threshold-type mechanism. It is clear that the sign of $\beta_{c}$ is determined by the sign of derivative $\mathrm{d} g_{1} / \mathrm{d} p$. Both experimental data and theoretical analysis of bubble and pore dynamics under pressure $[9,15]$ indicate that $\mathrm{d} g_{1} / \mathrm{d} p<0$, i.e., the voids become stiffer 
under increasing pressure. Thus, for materials containing only conventional bubbles or pores, the nonlinearity parameter $\beta=\beta_{c}$ should be positive, which is a well-known experimental fact. In contrast, the term $\beta_{t h}$ is negative since $\mathrm{d} R_{c r} / \mathrm{d} p<0$ (as clear from eq. (1)) and factor $g_{1}(R, p)$ is positive (since the sign of volume variation in eq. (6) is chosen positive for positive $\mathrm{d} p$ ). Depending on the current value of $R_{c r}(p)$ and the shape of the distribution function $n(R)$, the second term in eq. (9) can dominate ensuring the resultant negative coefficient of nonlinearity. Physically this means that with increasing pressure, the amount of buckled microspheres increases so strongly that their additional contribution to the material compressibility dominates over the opposite trend due to the stiffening of earlier buckled microspheres. Thus, in principle, the applied pressure can control both the sign and value of the nonlinearity parameter.

Ultimate value of the nonlinearity parameter of the threshold origin and its relation to the conventional "soft-rigid" mechanism. - Let us analyse eqs. (9)-(11) to estimate the ultimate value of the negative $\beta$, which is determined by the interplay of the conventional soft-rigid and threshold-type mechanisms. To reveal the similarity with the conventionally discussed nonlinearity of bubble-containing liquids $[7,8]$, we assume that the shear modulus of the matrix is sufficiently small to ensure that the gas pressure $P_{g}$ in the pores would control their deformability. Then using the quasistatic (i.e. far below the bubble resonance) approximation of the expression for adiabatic parameter $g_{1}$ obtained in $[9,15,16]$ and taking its isothermal derivative $\left(\mathrm{d} g_{1} / \mathrm{d} p\right)_{T}$ at $p=P_{g}$ we have

$$
g_{1}=\frac{1}{\gamma P_{g}}\left(\frac{4 \pi}{3} R^{3}\right), \quad\left(\frac{\mathrm{d} g_{1}}{\mathrm{~d} p}\right)_{T}=-\frac{\gamma+1}{\gamma P_{g}^{2}}\left(\frac{4 \pi}{3} R^{3}\right),
$$

where $\gamma$ is the adiabatic index of the gas. Note that the isothermal derivative $\left(\mathrm{d} g_{1} / \mathrm{d} p\right)_{T}$ in eq. (12) functionally differs from the quasistatic limit of formula (17) in [16] for a similar adiabatic parameter $g_{2}$ by one power of factor $\gamma$ in the denominator. Then eq. (10) for $\beta_{c}$ takes the form

$$
\beta_{c}=\gamma(\gamma+1) F(v, Q), F(v, Q)=\frac{v Q^{2}}{[1+v Q]^{2}},
$$

where $Q=K /\left(\gamma P_{g}\right) \gg 1$ is the ratio of compression moduli for the matrix and the gas. The factor $\gamma(\gamma+1)$ in eq. (13) appears due to the own conventional quadratic nonlinearity of the gas [7], whereas the factor $F(v, Q)$ describes the nonlinearity increase due to the locally strongly enhanced deformation of the soft inclusions (the soft-rigid mechanism). Note that eq. (13) agrees with the known expressions for the quadratic nonlinear parameter for liquids with bubbles [8] and elastic materials with soft inclusions [2,3]. As the volumetric fraction $v$ of soft inclusions increases, the parameter $F(v, Q)$ of the nonlinearity enhancement due to the sof-rigid mechanism also increases, reaches its maximum of $Q / 4 \gg 1$ at a small concentration $v=1 / Q \ll 1$ and then decreases again. Its maximum value can be rather high. For instance, for water with bubbles, the ratio $Q$ of the compressibility moduli for water and air is of the order of $10^{4}$. If the pore deformability is dominated by the hoop stiffness of its contour (i.e., by the matrix shear modulus $\mu$ ) rather than the gas pressure, then its relative softness is $Q \sim \lambda / \mu[9]$, which can also be rather high $\left(10^{3}-10^{5}\right)$ for incompressible elastic materials like soft plastics, biological tissues, etc. [17].

Let us now compare the above obtained estimates for $\beta_{c}$ with the ones for the term $\beta_{t h}$ (eq. (11)) reflecting the material nonlinearity related to the buckling. For the derivative $\mathrm{d} R_{c r} / \mathrm{d} p$, eq. (1) yields the following expression via the current value of the buckling radius:

$$
\mathrm{d} R_{c r} / \mathrm{d} p=-\frac{1}{2 \tilde{E}} R_{c r} \frac{R_{c r}^{2}}{t^{2}},
$$

where $\tilde{E}=2 E_{s} / \sqrt{3\left(1-\nu^{2}\right)}$. Recalling eqs. (11), (12) and (13) we can rewrite $\beta_{t h}$ as

$$
\beta_{t h} \approx-\frac{K}{2 \tilde{E}} \frac{R_{c r}^{2}}{t^{2}} \frac{4 \pi R_{c r}^{4} n\left(R_{c r}\right)}{3 Q v} F(Q, v) .
$$

In order to get an insight into the functional behaviour of the threshold-buckling nonlinearity, let us suppose that distribution $n(R)$ with maximum $n\left(R_{0}\right)$ is localized within a narrow interval $R_{0} \pm \Delta R, \Delta R \ll R_{0}$. We also rewrite eq. (2) for the volume fraction $v$ in a dimensionless form,

$$
v=\frac{4 \pi R_{c r}^{4} n\left(R_{c r}\right)}{3} \int_{1}^{\infty} \frac{n\left(\xi R_{c r}\right)}{n\left(R_{c r}\right)} \xi^{3} \mathrm{~d} \xi .
$$

Then for pressures $p$ such that $R_{c r}(p) \sim R_{0}$, the integral in the right-hand side of eq. (16) can be estimated as

$$
\int_{1}^{\infty} \frac{n\left(\xi R_{0}\right)}{n\left(R_{0}\right)} \xi^{3} \mathrm{~d} \xi \lesssim \int_{1}^{1+\Delta R / R_{0}} \xi^{3} \mathrm{~d} \xi \sim \frac{\Delta R}{R_{0}}
$$

Substituting this estimate into eq. (15) we have

$$
\left|\beta_{t h}\right| \geqslant \frac{K}{\tilde{E}} \frac{R^{2}}{t^{2}} \frac{1}{Q} \frac{R_{0}}{\Delta R} F(v, Q) \sim \frac{K}{\tilde{E}} \frac{R^{2}}{t^{2}} \frac{1}{Q} \frac{R_{0}}{\Delta R} \beta_{c} .
$$

Thus, assuming that $K / \tilde{E} \sim 1$ we conclude that the threshold-buckling mechanism can strongly dominate if $\left(R_{0}^{2} / t^{2}\right)\left(R_{0} / \Delta R\right) / Q \gg 1$. This requirement means that the shells are sufficiently thin $(t \ll R)$ and the distribution $n(R)$ is sufficiently narrow $\left(\Delta R \ll R_{0}\right)$. For example, for $R_{0} / \Delta R \sim 6-8, R / t \sim 200-500$ (which is realistic for $20-50 \mu \mathrm{m}$ radius and $0.1 \mu \mathrm{m}$ wall thickness of plastic microshells [11]) and typical compressibility contrast $Q \sim 10^{4}$ (for air-filled pores at the atmospheric pressure), we get parameter $\left(R_{0}^{2} / t^{2}\right)\left(R_{0} / \Delta R\right) / Q \sim 100-130$. Thus, even at the optimal concentration $v=1 / Q \ll 1$, where the factor $F(v, Q)$ of the conventional nonlinearity enhancement reaches its maximum value $Q / 4$ such that the 
corresponding positive $\beta_{c}$ is maximal, we conclude that $\left|\beta_{t h}\right| \gg \beta_{c}$ and $\beta \approx \beta_{t h}$. For the maximum value $F(v, Q)_{\max }=Q / 4$, eq. (18) then yields the estimate of the maximum $\beta$ dominated by the threshold mechanism:

$$
\beta \approx \beta_{t h} \approx-\frac{K}{4 \tilde{E}} \frac{R_{0}^{2}}{t^{2}} \frac{R}{\Delta R} .
$$

Assuming $K / \tilde{E} \approx 1, R / t \approx 500$, and $R / \Delta R \approx 8$ we obtain the estimate $|\beta| \approx 0.5 \times 10^{6}$. For narrower distributions $n(R)$, this value can evidently increase further up to several millions (but the strain range of the increased nonlinearity would correspondingly become narrower). In the case of the conventional soft-rigid mechanism, for which the maximum value of the nonlinearity is controlled by the relative softness $Q$ of the soft fraction, comparable values of $\beta_{c}$ assume unrealistically high values of $Q \gtrsim 10^{6}$.

Regarding the obtained very high estimates of $|\beta|$ one should bear in mind that the nonlinear correction was assumed to remain smaller than the linear term in the equation of material state. Therefore, the considered strains $\varepsilon$ are limited by the condition $|\varepsilon \beta|<1$. Nevertheless, it seems possible to create a highly strain-sensitive material in which strains $\varepsilon \sim 10^{-6}$ can produce variations in the elastic modulus comparable with its initial value.

It should also be noted that while a distribution of shell-wall thickness could affect the above considerations, the main qualitative conclusions remain valid. Evidently, the simplest case is when the distribution function can be factorized, $n(t, R)=n(t) n(R)$, such that the above results obtained for fixed thickness $t$ can be averaged over $n(t)$.

Experimental. - To demonstrate the strong dominance of the threshold-type mechanism over the conventional soft-rigid mechanism, we chose gelatin as the matrix material, although there are available numerous soft plastics exhibiting high ratios $\lambda / \mu$ (and, correspondingly, Poisson's ratios close to 0.5 ). To play a role of encapsulated pores, we chose commercially produced microspheres (091WE40 d24 [11]). According to the producer's specifications, their typical wall thickness is $t \sim 0.1 \mu \mathrm{m}$ and the distribution function over radii is bell shaped with center at $R_{0} \sim 50 \mu \mathrm{m}$ and width $2 \Delta R / R_{0} \sim 30 \%$, which corresponds to the assumptions used above. Assuming that the compressibility of the buckled microspheres is mostly determined by the pressure of the filling gas, the optimal concentration of the inclusions was expected to be on the order of $10^{-4}$. In view of the difficulty to prepare a homogeneous mixture with such a small volume content of the inclusions, we used higher concentrations $\sim(1-2) \times$ $10^{-3}$. However, even then each cubic millimeter contained only 2-4 microspheres. The prepared mixture was gelled in a stainless-steel tube $50 \mathrm{~cm}$ in length with inner and outer diameters of $D_{i}=9.5 \mathrm{~mm}$ and $D_{o}=12 \mathrm{~mm}$. Another identical tube was filled with pure gelatin and served as a reference sample. The ends of the tubes were closed by aluminium cylindrical plugs (9 $\mathrm{mm}$ in diameter) sealed by a thin rubber ring each. Small $(1 \mathrm{~cm}$ in diameter)

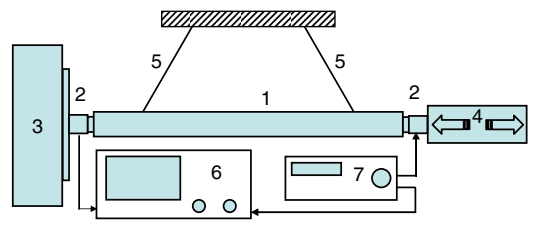

Fig. 1: (Colour on-line) Sketch of the setup: 1 is the gelatinfilled steel tube, 2 is the pair of transducers mounted on plugs, 3 is the electronic balance, 4 is the microscrew positioning system, 5 are supporting threads, 6 is oscilloscope, and 7 is the signal generator.

$P$-wave piezo-transducers were glued on the plugs. The tubes were horizontally suspended by supporting threads as shown in fig. 1. One end of the tube was abutted on the surface of a microscrew positioning system and the other one touched the vertically oriented surface of an electronic scale. The measured compressing force could be readily translated into the applied pressure $p$. Under the used loads up to $1-4 \mathrm{~N}$ (corresponding to pressures $(1-5) \times 10^{4} \mathrm{~Pa}$ ), the relatively low stiffness of the balance ensured displacements on the order of tens of micrometers, which could be easily controlled by the conventional microscrew. The actual axial deformation of the material inside the tube remained much smaller, on the micrometer scale, and corresponded to strains $\lesssim 10^{-5}$, which was estimated as the ratio $p / K_{\text {eff }}$, (where the effective bulk modulus $K_{\text {eff }}=\rho c^{2}$ was determined by measuring the wave velocity $c$ ). Note also that under micrometer-scale axial deformations used, the gelatin remained adhered to the tube walls. It can readily be shown that beyond the characteristic scale $L_{s c} \sim\left(D_{i} / 2\right)(\lambda / \mu)^{1 / 2}$, the compressional stress (applied from the both ends of the tube) could be screened by the resultant shear force between the wall and gelatin. However, for $\lambda / \mu \gtrsim 10^{4}-10^{5}$ typical of gelatin [17], $L_{s c}$ is at least $\gtrsim 25-50 \mathrm{~cm}$, such that the inhomogeneity of the pressure distribution along the tube should not be significant. In any case the screening effect could only increase the nonlinearity estimate obtained below in the assumption of homogeneous compressional strain.

We recall (see, e.g., $[1,2,7]$ ) that even at relatively small concentrations comparable with that in our experiment, the conventional shell-less bubbles or pores can strongly reduce the modulus of liquids and incompressible elastic materials. The first striking feature of the gelatin with encapsulated pores is that the observed wave velocity in the nonloaded sample is only slightly (within $5-10 \%$ ) reduced compared to the reference sample. This confirms that the presence of apparently "weak" shells can very significantly reduce the compressibility of the pores before the shell buckling. Another striking feature was that under loading to moderate strains $\sim 10^{-5}$, the velocity of the propagated weak-amplitude pulses of the probe wave (with estimated strains $\sim 10^{-8}$ ) in the microinhomogeneous sample were reduced by $100-400 \mathrm{~m} / \mathrm{s}$. In contrast, for the 


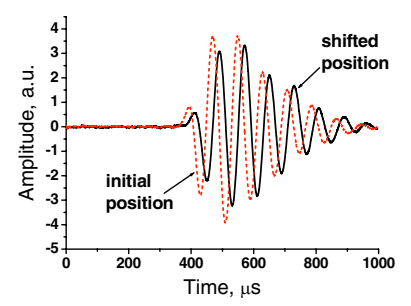

Fig. 2: (Colour on-line) Example of the pulse shift (corresponding to the velocity decrease from $1380 \mathrm{~m} / \mathrm{s}$ to $1290 \mathrm{~m} / \mathrm{s}$ ) in the microinhomogeneous sample compressed to strain $7 \cdot 10^{-6}$. In the measurements, bursts with $6-14 \mathrm{kHz}$ carrier frequency were used.

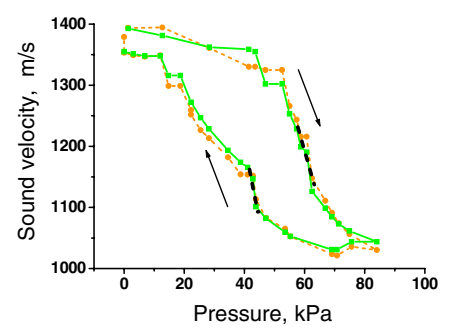

Fig. 3: (Colour on-line) Example of the pressure dependence of the sound velocity. The solid line corresponds to measuring the shift of the first minimum of the pulse; the dashed line is obtained by measuring the phase shift in the maximum of the pulse spectrum. Arrows show loading-unloading of the sample.

reference sample, the velocity variation was unmeasurable. Examples of the corresponding oscillograms observed for probe-wave pulses are shown in fig. 2. Figure 3 shows an example of the dependence of the sound velocity upon the applied pressure. The two curves correspond to two different methods of measuring the wave velocity: via the shift of the first minimum in the pulse (solid line) and the phase shift in the maximum of the pulse spectrum (dashed line). The curves are in a good agreement and indicate up to a $40 \%$ velocity variation due to sequential buckling and then restoration of the shells in the pressure range applied. The reason for the observed hysteresis in the velocity restoration is physically clear: the buckled shells evidently restore their shape when under unloading, the pressure falls by a finite value below the buckling threshold. However, a detailed discussion of the hysteresis is beyond the scope of the present paper. Here, we would like to point out that the derivative of the velocity pressure dependence (at the segments shown in thick dashed line) indicates the quadratic nonlinearity parameter $|\beta|=\left|\mathrm{d} K_{\text {eff }} / \mathrm{d} p\right| \sim$ $(0.5-1) \cdot 10^{5}$. This value can be increased by fine tuning the parameters of the microspheres and their concentration. To the best of our knowledge, the obtained value of the nonlinearity parameter is an order of magniture higher than reliably determined genuine (not "equivalent" as discussed in the introduction) values of $B / A \sim(5-10) \cdot 10^{3}$ in bubbly liquids and porous incompressible materials $[14,17,18]$. We emphasize that the nonlinearity observed in our experiments is nonresonant, whereas some previous estimations were obtained using dynamic resonance effects which considerably enhance nonlinearity.
The presented analysis shows that the threshold-type behaviour of the soft inclusions can radically modify predictions based on the conventionally discussed softrigid mechanism. Evidently these features can be of interest for bio-medical diagnostic applications, where the encapsulated "bubbles" are normally considered as merely a more stable form of conventional bubbles. From the physicist's viewpoint, such materials with controllable giant nonlinearity open unique experimental possibilities for studying wave transformations in media with elastic nonlinearity and hysteretic properties.

$$
* * *
$$

The study was supported by ARC Discovery Grants DP0771044 (AD) and DP0773839 (EP) as well as by a start-up grant of the UWA Faculty of Engineering, Computing and Mathematics and School of Mechanical Engineering (EP). The research was conducted during a visit of VYuZ to UWA supported by the Gledden Foundation.

\section{REFERENCES}

[1] Naugolnykh K. and Ostrovsky L., Nonlinear Wave Processes in Acoustics (Cambridge University Press, New York) 1998.

[2] Zaitsev V., Acoust. Lett., 19 (1996) 171.

[3] Belyaeva I. Yu. and Zaitsev V. Yu., Acoust. Phys., 43 (1997) 594.

[4] Johnson P. and Sutin A., J. Acoust. Soc. Am., 117 (2005) 124.

[5] Tournat V., Zaitsev V., Gusev V., Nazarov V., Bequin P. and Castagnede B., Phys. Rev. Lett., 92 (2004) 085502.

[6] Tournat V., Gusev V., Zaitsev V. and Castagnede B., Europhys. Lett., 66 (2004) 798.

[7] Rudenko O. V. and Soluyan S. I., Theoretical Foundations of Nonlinear Acoustics (Consultants Bureau, New York) 1977.

[8] Apfel R. E., J. Acoust. Soc. Am., 74 (1983) 1866.

[9] Ostrovsky L. A., Sov. Phys. Acoust., 34 (1988) 523.

[10] http://en.wikipedia.org/wiki/Contrast-enhanced ultrasound.

[11] Website of the product: www.expancel.com.

[12] Landau L. D. and Lifshitz E. M., Theory of Elasticity (Pergamon, New York) 1959.

[13] Kollár L., in Buckling of Shells, edited by RAM E. (Springer, Berlin, Heidelberg, New York) 1982, pp. 401425.

[14] Trivett D. H., Pincon H. and Rogers P. H., J. Acoust. Soc. Am., 119 (2006) 3610.

[15] Emelianov S., Hamilton M., Ilinskit Yu. and Zabolotskaya E., J. Acoust. Soc. Am., 115 (2004) 581.

[16] Qin B., Chen J. and Cheng J., Acoust. Phys., 52 (2006) 418.

[17] Belyaeva I. Yu. and Timanin E. M., Sov. Phys. Acoust., 37 (1991) 533.

[18] Gong X. F., Ye S. G., Zhang D., Feng S. S., Zhang R. Q., Wang R. T., Xu Z. Z., Liu L. M. and Ha K. L., J. Acoust. Soc. Am., 103 (1998) 2960. 